\title{
OPEN Effects of physical exercise on macular vessel density and choroidal thickness in children
}

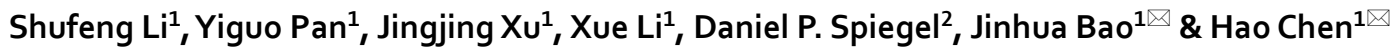

We used swept-source (SS) optical coherence tomography (OCT) and OCT angiography (OCTA) to investigate the effects of moderate physical exercise on retinal and choroidal vessel densities (VDs) and thicknesses in children. One eye in each of 40 myopic children (mean age, 11.70 years) and 18 emmetropic children (mean age, 11.06 years) were included. SS-OCT $6 \times 6-\mathrm{mm}$ radial scans and SS-OCTA $3 \times 3-\mathrm{mm}$ images were centered on the macula. Heart rate (HR), systolic and diastolic blood pressure, and intraocular pressure (IOP) were recorded before and immediately after a 20-min stationary cycling exercise and after a 30-min rest. The subfoveal choroidal thickness (SFCT), choroidal thickness (CT), and VD at the superficial and deep retinal layers, choriocapillaris, and deeper choroidal vessels were determined. SFCT and CT were significantly lower at all locations immediately after exercise $(p<0.001)$ and did not fully recover after rest $(p<0.05)$. VD was lower in the deep retinal layer after exercise $(p=0.02)$ and higher in the superficial layer after rest $(p=0.03)$ in myopic eyes while it was higher in the superficial $(p<0.01)$ and deep layer $(p<0.01)$ after rest in emmetropic eyes. No significant exercise-related changes in the superficial retinal VD, choroidal VD, or IOP were observed. $\Delta C T \%$ and $\triangle$ SFCT\% were significantly correlated with increases in HR in myopic group $(p=0.04$ and $p=0.03$, respectively). Exercise increased retinal VD after rest in emmetropic eyes, and caused significant CT thinning that lasted for at least $30 \mathrm{~min}$ in both emmetropic and myopic eyes.
\end{abstract}

Physical exercise has positive effects in children ${ }^{1,2}$. The benefits of exercise can be attributed to several mechanisms, including increased cardiorespiratory fitness and the strategic redistribution of blood flow that occurs through vasodilatation of the heart and skeletal muscles and/or vasoconstriction of the skin and splanchnic tissues ${ }^{3}$. Proteins, peptides, enzymes, and metabolites released from one organ can affect the metabolism of other organs as the blood flow becomes redistributed during exercise ${ }^{4}$. While the effects of physical exercise have been studied in many organs of both adults and children, how it affects the eyes in children is relatively unknown. The retina and choroid are densely vascularized, and appropriate levels of ocular blood flow are indispensable to normal visual performance ${ }^{5}$. Given that exercise changes the cardiovascular system throughout the body, changes in blood pressure (BP) and heart rate (HR) are likely to affect the retina and choroid.

Some previous studies have investigated the associations between exercise-induced alterations in the systemic circulation and changes in the retina and choroid in adults, but the results have been controversial ${ }^{6-10}$. Alnawaiseh et al. ${ }^{10}$ reported that peripapillary and parafoveal flow densities decreased significantly after exercise. Vo Kim et al. ${ }^{6}$ also reported that the vessel density (VD) at the level of the retinal superficial layer, from $3 \mu \mathrm{m}$ below the internal limiting membrane (ILM) to $15 \mu \mathrm{m}$ below the inner plexiform layer (IPL), were decreased after exercise while the fractal dimension in the retinal deep layer, from 15 to $70 \mu \mathrm{m}$ below the IPL, were significantly increased. Hayashi et al. ${ }^{7}$ found that the blood flow and vascular conductance of retinal arterioles remained stable while ocular blood flow increased in the retina and choroid during physical exercise. Changes reported for choroidal thickness (CT) associated with exercise were inconsistent among previous studies. CT remained stable in most of these reports ${ }^{11,12}$ but increased in others ${ }^{13}$.

Although most epidemiological studies have demonstrated that the benefits for children of outdoor activity are derived from exposure to light, the effects of exercise on eye structure and function have not been well studied $^{14}$. The aim of our study was to evaluate the impact of exercise-induced changes in retinal VD (RVD), choroidal VD (CVD), and CT in children. We used swept-source optical coherence tomography angiography (SS-OCTA) and swept-source optical coherence tomography (SS-OCT) to measure the effects of exercise on

${ }^{1}$ Eye Hospital, School of Ophthalmology and Optometry, Wenzhou Medical University, 270 Xueyuan Road, Wenzhou 325027, Zhejiang, China. ${ }^{2}$ R\&D Vision Sciences AMERA, Essilor International, Singapore, Singapore. ${ }^{\boxplus}$ email: baojessie@163.com; chenhao@mail.eye.ac.cn 
A

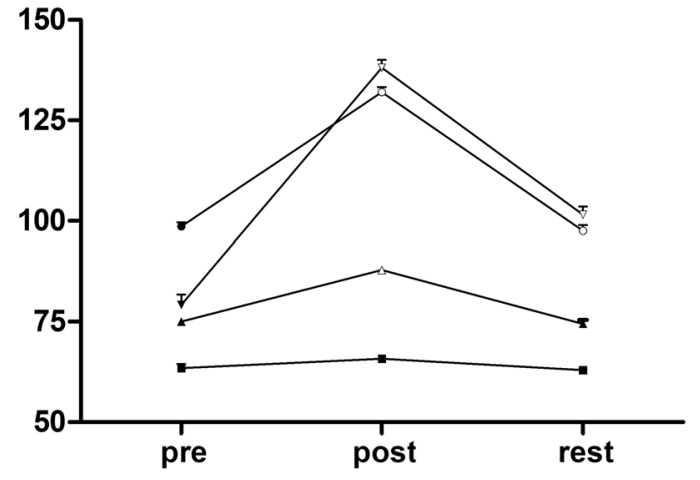

B

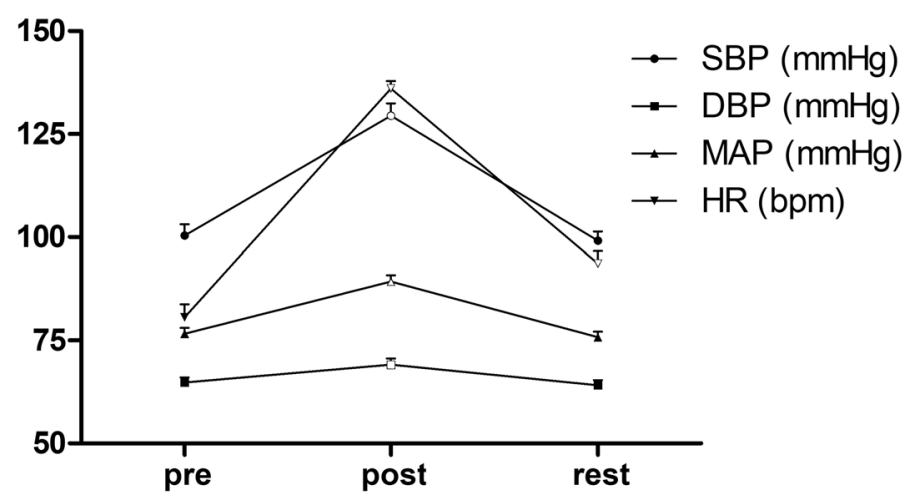

Figure 1. Changes in systemic dynamic parameters among measurements obtained before the 20 min of cycling exercise, immediately after the exercise, and after the 30-min rest period. (A) Systemic dynamic parameters of myopic group. (B) Systemic dynamic parameters of emmetropic group. The error bars represent the standard error of the mean, all compared with the data before exercise (hollow symbols represent $\mathrm{p}<0.001$ vs. pre-exercise). $S B P$ systolic blood pressure; $D B P$ diastolic blood pressure, $M A P$ mean arterial pressure, $H R$ heart rate.

RVD, CVD, and CT, and we evaluated correlations between these ocular parameters and systemic physiological parameters.

\section{Results}

Fifty-eight eyes from 58 children were enrolled in this study (ages 9-13 years): 40 myopic eyes (mean spherical equivalent (SE): $-3.27 \pm 1.16$ diopter (D)), 18 emmetropic eyes (mean SE: $0.03 \pm 0.31 \mathrm{D}$ ). All of the subjects were free of any ocular or systemic disease and were not taking any medication.

Changes in systemic hemodynamic parameters. In myopic group, the mean systolic blood pressure (SBP), mean arterial pressure (MAP), and HR were significantly higher after 20 min of exercise than before, changing from $98.65 \pm 8.16$ to $132.0 \pm 9.77 \mathrm{mmHg}$, from $75.03 \pm 5.70$ to $87.85 \pm 6.00 \mathrm{mmHg}$, and from $79.23 \pm 12.45$ to $138.15 \pm 11.19 \mathrm{bpm}$, respectively (all $p<0.001$, Fig. 1A). After a 30 -min rest, these values returned close to the pre-exercise levels, except for HR, $101.58 \pm 10.15 \mathrm{bpm}$, which was significantly decreased but still higher than at baseline $(p<0.001)$. In addition, HR increased to more than $66 \%$ of the age-dependent maximal HR (estimated as $220-$ age $^{15}$ ). The IOP after exercise, $17.25 \pm 2.75 \mathrm{mmHg}$, and after the rest period, $17.83 \pm 2.73 \mathrm{mmHg}$, were not significantly different from the pre-exercise value, $17.23 \pm 2.03 \mathrm{mmHg}(p=0.947$ and $p=0.106$, respectively). The control group completed the same exercise protocol with similar changes (Fig. 1B).

Changes in SS-OCT and SS-OCTA parameters. The overall CT was significantly reduced after exercise in both myopic and emmetropic eyes, changing from $227.45 \pm 41.76$ to $210.53 \pm 39.46 \mu \mathrm{m}$ in myopic eyes and from $271.14 \pm 54.89 \mu \mathrm{m}$ to $261.86 \pm 53.37$ in emmetropic eyes (both $p<0.001$ ). After rest, the thickness partially recovered but still remained thinner than before exercise, $218.40 \pm 40.08 \mu \mathrm{m}$ in myopic eyes $(p<0.01)$ and $265.65 \pm 52.31 \mu \mathrm{m}$ in emmetropic eyes $(p<0.05$, Fig. 2$)$. Similarly, subfoveal choroidal thickness (SFCT) was significantly lower after exercise than before, changing from $226.60 \pm 47.08$ to $212.33 \pm 45.22 \mu \mathrm{m}$ in myopic eyes $(p<0.001)$, from $277.41 \pm 58.75$ to $265.43 \pm 54.86 \mu \mathrm{m}$ in emmetropic eyes. The SFCT partially recovered after rest but was still thinner than that before exercise, $217.70 \pm 45.18 \mu \mathrm{m}$ in myopic eyes and $271.54 \pm 57.03 \mu \mathrm{m}$ in emmetropic eyes $(p<0.05)$. Significantly, the changes in CT\% of myopic eyes were larger than that of emmetropic eyes $(-7.45 \pm 3.32 \%$ vs. $-3.77 \pm 1.58 \%, p=0.007)$.

In the superficial layer of the retina in myopic eyes, the pre-exercise overall and parafoveal VD values, $43.35 \pm 1.63 \%$ and $48.88 \pm 1.57 \%$ (Table 1$)$, did not change significantly after the exercise period $(p=0.46$ and $p=0.67$ respectively). However, after the 30 -min rest period, the parafoveal VD, $49.20 \pm 1.50 \%$, were higher than the pre-exercise values $(p=0.03)$. There were no significant changes in the superficial foveal VD during the study. In the deep layer of the retina in myopic eyes, the overall VD and parafoveal VD values was significantly lower after exercise than before exercise, changing from $44.20 \pm 1.41 \%$ and $50.90 \pm 1.71 \%$ to $43.87 \pm 1.55 \%$ and $50.54 \pm 1.75 \%$ ( $p=0.02$ and $p=0.03$ respectively), and they returned approximately to the pre-exercise level after rest (Table 1). The foveal VDs of the deep layer of the retina in myopic eyes remained stable during the study. In emmetropic eyes, after the 30-min rest, the overall and parafoveal VD values in both the superficial and deep layers were higher than the pre-exercise values $(p<0.01$ and $p=0.02$ in superficial layer, both $p<0.01$ in the deep layer, respectively).

There were no significant CVD differences in slabs of the choriocapillaris, inner choroid, mid-choroid, and outer choroid during the study in both myopic and emmetropic eyes (Fig. 3A-D, A1-D1). However, the CVD in slabs of the mid-choroid tended to decrease after the exercise in myopic eyes, changing from $69.40 \pm 2.47$ to $68.83 \pm 2.31 \%(p=0.07$, Fig. $3 C)$. 

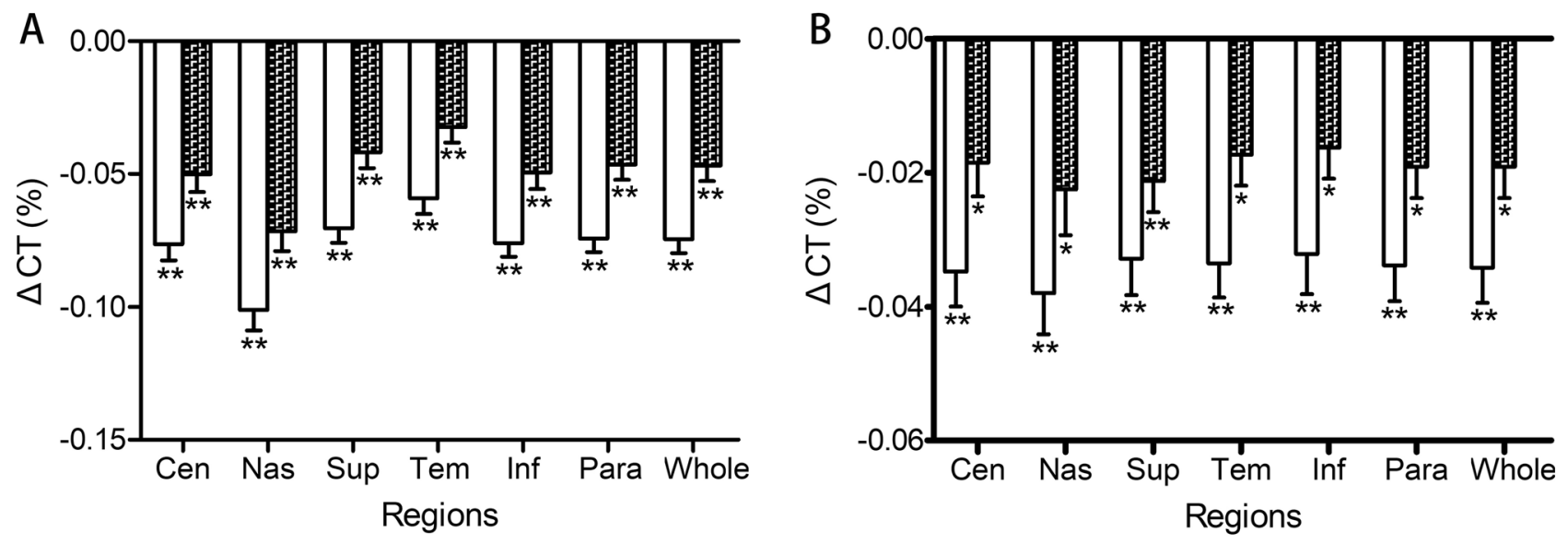

Figure 2. Changes in CT\% at different locations immediately after exercise and after rest. (A) Changes of CT\% at different locations of myopic eyes. (B) Changes of CT\% at different locations of emmetropic eyes. The error bars represent the standard error of the mean $\left({ }^{*}, p<0.05 ;{ }^{* *}, p<0.001\right.$ vs. pre-exercise, respectively). $C T$ choroidal thickness; Cen central region; Nas nasal; Sup superior; Tem temporal; Inf inferior represents the mean data of inner and outer regions. Para, parafoveal region; Whole, whole region.

\begin{tabular}{|c|c|c|c|c|c|}
\hline & Variables & $\begin{array}{l}\text { Pre-exercise } \\
(95 \% \text { CI })\end{array}$ & \begin{tabular}{|l} 
Post-exercise \\
$(95 \%$ CI $)$
\end{tabular} & $\begin{array}{l}\text { Rest } \\
(95 \% \mathrm{CI})\end{array}$ & $\begin{array}{l}P \text { Value } \\
\text { (post-pre/rest-pre) }\end{array}$ \\
\hline \multirow{6}{*}{$\begin{array}{l}\text { Myopia } \\
(\mathrm{N}=40)\end{array}$} & Superficial overall RVD(\%) & $\begin{array}{l}43.35 \pm 1.63 \\
(42.83-43.87)\end{array}$ & $\begin{array}{l}43.25 \pm 1.50 \\
(42.77-43.73)\end{array}$ & $\begin{array}{l}43.63 \pm 1.45 \\
(43.17-44.09)\end{array}$ & $0.462 / 0.061$ \\
\hline & Superficial parafoveal RVD (\%) & $\begin{array}{l}48.88 \pm 1.57 \\
(48.37-49.38)\end{array}$ & $\begin{array}{l}48.82 \pm 1.53 \\
(48.33-49.31)\end{array}$ & $\begin{array}{l}49.20 \pm 1.50 \\
(48.72-49.68)\end{array}$ & $0.668 / \mathbf{0 . 0 3 0}$ \\
\hline & Superficial foveal RVD (\%) & $\begin{array}{l}21.24 \pm 4.99 \\
(19.65-22.84)\end{array}$ & $\begin{array}{l}20.97 \pm 5.05 \\
(19.35-22.58)\end{array}$ & $\begin{array}{l}21.35 \pm 5.00 \\
(19.75-22.95)\end{array}$ & $0.405 / 0.744$ \\
\hline & Deep overall RVD (\%) & $\begin{array}{l}44.20 \pm 1.41 \\
(43.80-44.68)\end{array}$ & $\begin{array}{l}43.87 \pm 1.55 \\
(43.52-44.51)\end{array}$ & $\begin{array}{l}44.28 \pm 1.52 \\
(43.88-44.76)\end{array}$ & $\mathbf{0 . 0 2 2} / 0.568$ \\
\hline & Deep parafoveal RVD (\%) & $\begin{array}{l}50.90 \pm 1.71 \\
(50.42-51.59)\end{array}$ & $\begin{array}{l}50.54 \pm 1.75 \\
(50.01-51.32)\end{array}$ & $\begin{array}{l}51.03 \pm 1.68 \\
(50.58-51.58)\end{array}$ & $\mathbf{0 . 0 3 1} / 0.517$ \\
\hline & Deep foveal RVD (\%) & $\begin{array}{l}17.37 \pm 4.59 \\
(15.91-19.00)\end{array}$ & $\begin{array}{l}17.19 \pm 4.21 \\
(16.12-18.59)\end{array}$ & $\begin{array}{l}17.30 \pm 4.30 \\
(15.92-18.92)\end{array}$ & $0.619 / 0.865$ \\
\hline \multirow{6}{*}{$\begin{array}{l}\text { Control } \\
(\mathrm{N}=18)\end{array}$} & Superficial overall RVD(\%) & $\begin{array}{l}41.66 \pm 1.19 \\
(41.07-42.25) \\
\end{array}$ & $\begin{array}{l}41.78 \pm 1.27 \\
(41.15-42.42) \\
\end{array}$ & $\begin{array}{l}42.19 \pm 1.34 \\
(41.53-42.86)\end{array}$ & $0.399 / \mathbf{0 . 0 0 8}$ \\
\hline & Superficial parafoveal RVD (\%) & $\begin{array}{l}47.04 \pm 1.32 \\
(46.38-47.70)\end{array}$ & $\begin{array}{l}46.19 \pm 1.77 \\
(45.31-47.07)\end{array}$ & $\begin{array}{l}47.56 \pm 1.44 \\
(46.84-48.27)\end{array}$ & $0.129 / \mathbf{0 . 0 1 5}$ \\
\hline & Superficial foveal RVD (\%) & $\begin{array}{l}20.14 \pm 3.87 \\
(18.21-22.06)\end{array}$ & $\begin{array}{l}20.72 \pm 4.17 \\
(18.64-22.79)\end{array}$ & $\begin{array}{l}20.73 \pm 4.22 \\
(18.64-22.83)\end{array}$ & $0.036 / 0.036$ \\
\hline & Deep overall RVD (\%) & $\begin{array}{l}42.19 \pm 1.63 \\
(41.38-43.00)\end{array}$ & $\begin{array}{l}42.67 \pm 2.01 \\
(41.67-43.67)\end{array}$ & $\begin{array}{l}43.08 \pm 1.73 \\
(42.22-43.95)\end{array}$ & $0.054 / \mathbf{0 . 0 0 2}$ \\
\hline & Deep parafoveal RVD (\%) & $\begin{array}{l}48.84 \pm 1.59 \\
(48.05-49.63)\end{array}$ & $\begin{array}{l}49.38 \pm 2.06 \\
(48.36-50.41)\end{array}$ & $\begin{array}{l}49.96 \pm 1.81 \\
(49.06-50.86)\end{array}$ & $0.072 / \mathbf{0 . 0 0 3}$ \\
\hline & Deep foveal RVD (\%) & $\begin{array}{l}15.56 \pm 3.72 \\
(13.71-17.41)\end{array}$ & $\begin{array}{l}15.80 \pm 3.71 \\
(13.96-17.65)\end{array}$ & $\begin{array}{l}15.57 \pm 4.35 \\
(13.41-17.74)\end{array}$ & $0.125 / 0.977$ \\
\hline
\end{tabular}

Table 1. Changes in RVD in the superficial and deep layers of myopic and emmetropic eyes before exercise, after $20 \mathrm{~min}$ of exercise, and after $30 \mathrm{~min}$ of rest. RVD retinal vessel density, CI confidence interval, post-pre comparisons by paired $t$ tests for post-exercise vs. pre-exercise, rest-pre: comparisons by paired $t$ tests for postrest vs. pre-exercise. Bold values indicate statistical significance: $p<0.05$.

Correlations among SS-OCT, SS-OCTA, and hemodynamic parameters. Changes in HR were negatively correlated with changes in CT\% (Pearson's correlation $r=-0.327, p=0.04$, Fig. $4 \mathrm{~A}$ ) and changes in SFCT\% (Pearson's correlation $\mathrm{r}=-0.345, p=0.03$, Fig. 4B) in myopic eyes. This correlation was not observed in emmetropic eyes. There were no significant correlations between $\triangle \mathrm{HR}$ and other SS-OCTA parameters in both myopic and emmetropic eyes. No significant correlations were detected among $\Delta \mathrm{SBP}, \Delta \mathrm{CT} \%$, and other SS-OCTA parameters. 
A

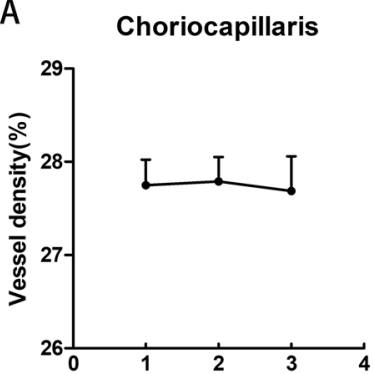

C

Mid choroid

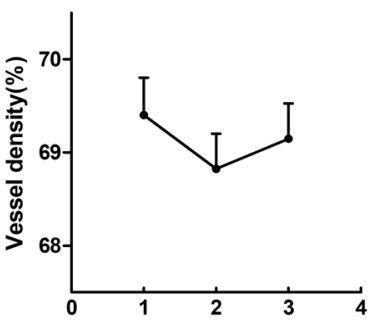

B

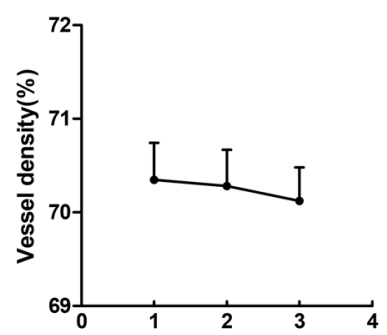

D

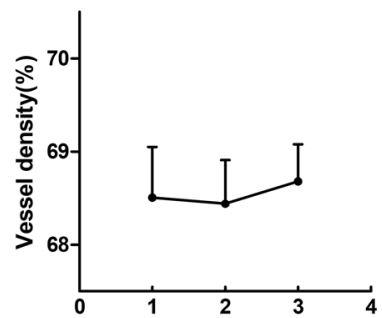

A1

Choriocapillaris

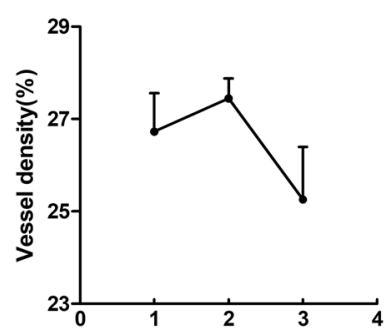

C1

Mid choroid

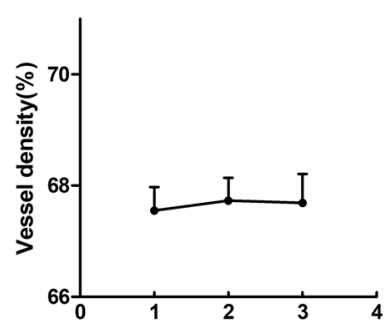

B1

Inner choroid

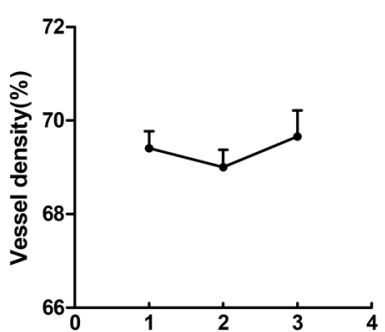

D1

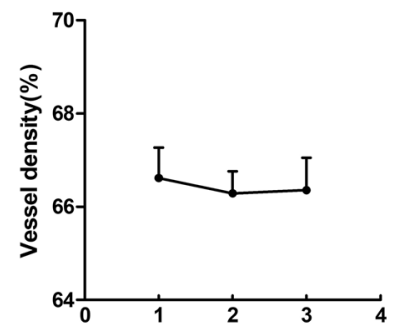

Figure 3. Changes in choroidal vessel densities (VD\%) of myopic and emmetropic eyes in the choriocapillaris (A, A1), inner choroid (B, B1), mid-choroid (C, C1), and outer choroid (D, D1) across measurements obtained before exercise, immediately after exercise, and after the 30-min rest period. The error bars represent the standard error of the mean.

A

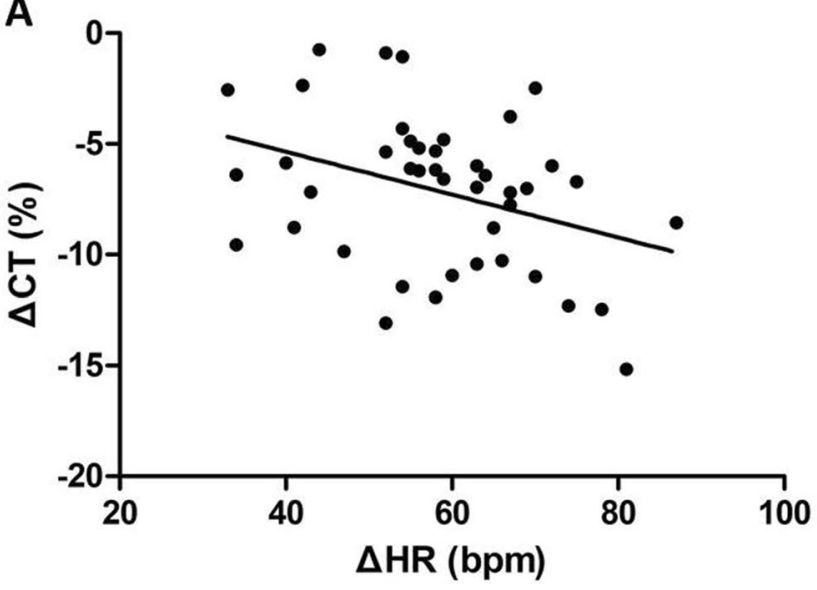

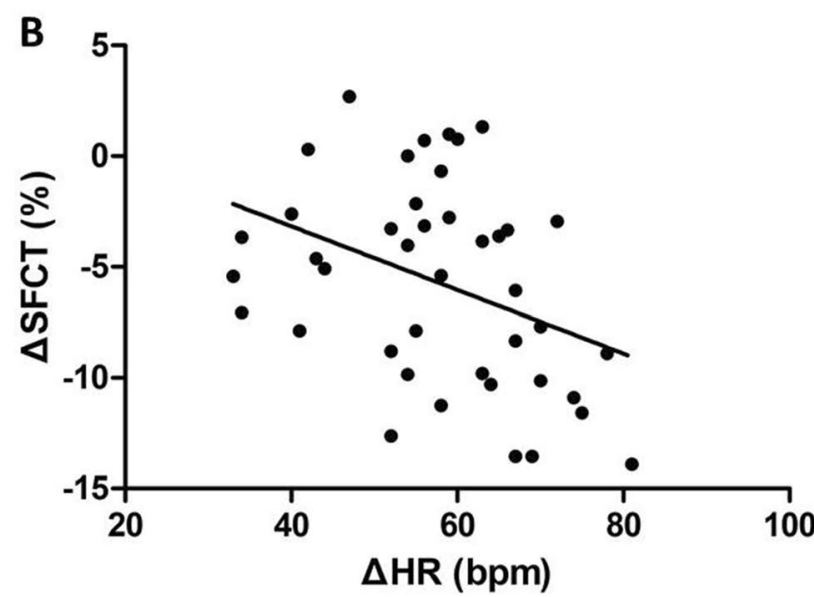

Figure 4. Scatter plot of $\triangle \mathrm{CT} \%$ against $\Delta \mathrm{HR}(\mathbf{A})$ and scatter plot of $\Delta \mathrm{SFCT} \%$ against $\Delta \mathrm{HR}(\mathbf{B})$ of myopic eyes. $C T$ mean choroidal thickness; SFCT subfoveal choroidal thickness; HR heart rate.

\section{Discussion}

In this study, we evaluated the effects of physical exercise on the CT and VD in the eyes of myopic and emmetropic children 9-13 years old, and we evaluated correlations among systemic dynamic parameters and the ocular changes. First, we observed that CT was significantly decreased after exercise in both myopic and emmetropic eyes, and this change continued to be significant after a 30-min rest period following the exercise. This finding differs from results reported for adults in which the CT was stable or even increased after exercise ${ }^{11,12}$. These inconsistencies may stem from the influence of the subjects' health status, age, and exercise intensity. Alwassia et al. ${ }^{11}$ recruited patients who underwent cardiac stress testing, while Kinoshita et al. ${ }^{12}$ used a mild exercise protocol that may have had a limited effect on the choroid. We found that the HR was more than $66 \%$ of the age-dependent maximal HR immediately after exercise, indicating that the exercise intensity in this study was moderate or even more than moderate ${ }^{16}$. Our results showed a significant negative correlation of $\Delta H R$ with both $\triangle \mathrm{CT} \%$ and $\triangle \mathrm{SFCT} \%$ after exercise in myopic eyes, suggesting that the CT changes of myopic eyes may have been affected by exercise intensity. However, this correlation was not observed in the emmetropic eyes. In addition, the changes in CT\% in myopic eyes were significantly larger than that in emmetropic eyes after completing the same exercise protocol. These different responses to exercise in myopic and emmetropic eyes may indicate differences in structure or function between these eyes. 
The contractility of vascular and nonvascular smooth muscles and the elasticity of elastic fibers differ between children and adults, and aging can lead to arterial stiffening ${ }^{17}$. The choroid mainly consists of blood vessels and stroma, which contain nonvascular smooth muscle cells and elastic fibers ${ }^{18}$. In our study, we found that CT was decreased after exercise while CVD was relatively stable in both myopic and emmetropic eyes. The contraction of vascular and nonvascular smooth muscle cells induced by sympathetic excitation during exercise may be one of the reasons for the reduced $\mathrm{CT}^{19}$. In addition, dehydration induced by sweating may cause water in the matrix to enter blood vessels, thus compensating for the decrease in blood volume ${ }^{20}$. Considering all of these factors, we propose that $20 \mathrm{~min}$ of moderate physical exercise by children may lead to a significant decrease in CT that lasts for at least $30 \mathrm{~min}$, while the water in matrix entering vessels maintained the CVD relatively stable. If the exercise time prolonged, whether CVD will continue to be stable with sweat loss is unknown. Previous studies have found that decreased CVD in myopic animal models is harmful to the retina and sclera, and that decreases in CT are related to the degree of myopia ${ }^{21,22}$. Therefore, whether or not a cumulative effect of exercise-induced choroidal thinning and decreased VD exists and whether or not this effect is harmful to the retina and sclera in children are questions requiring further study.

Our second important finding was that immediately after exercise, RVD was significantly decreased in the deep retinal layer in myopic eyes. This result was consistent with those presented in studies performed in adults in which macular perfusion decreased after exercise ${ }^{10}$. Ikemura et al. ${ }^{20}$ found that the blood flow in the internal carotid artery was significantly lower after exercise. They proposed that this phenomenon represented a reduction in the conductance index resulting from increases in blood viscosity, sympathetic activity, and the mean arterial partial pressure of $\mathrm{CO}_{2}\left(\mathrm{PaCO}_{2}\right)$ levels. In addition, the redistribution of blood to muscles and skin during exercise may also be involved in reducing the blood flow in the internal carotid artery, which may then influence fundus VD. Although autoregulatory mechanisms in the retina tend to maintain a constant blood flow over a wide range of perfusion pressures, blood flow can decrease beyond the compensation range ${ }^{23,24}$. Previous studies in adults showed that macular perfusion decreases in the superficial retinal layer after exercise, and that VD remains stable in the deep layer ${ }^{6,20}$. This discrepancy between the variation trend of RVD in superficial and deep layers after exercise may be attributable to differences in the elasticity and hardness of the fundus vessels between children and adults, which may lead to differences in autoregulatory ability ${ }^{17}$. As for the difference of RVD in deep layer between myopic and emmetropic eyes, it may also come from the different autoregulation capacity. Better vascular elasticity in emmetropic eyes results in faster RVD recovery after exercise before we can detect the fluctuation. Further animal studies may be needed to determine whether there is a difference in elasticity between retinal vessels in myopic and emmetropic eyes.

Researchers investigating functional hyperemia in the brain have suggested that arterioles are the primary sites of blood flow regulation ${ }^{25}$. In our study, when changes in BP were beyond the autoregulatory capacity of the retina, the RVD remained stable in the superficial layer while it decreased in the deep layer in myopic eyes. Based on this phenomenon, we infer that the arteries in the superficial layer, but not the deep layer, which consists of capillaries, tended to maintain a constant blood flow. Interestingly, the parafoveal RVD in the superficial layer in myopic eyes and RVD of all regions and layers in emmetropic eyes after the 30-min rest period were higher than the pre-exercise values. This effect may be induced by the recovery of $\mathrm{PaCO}_{2}$ due to breathing stabilization and discontinued hyperventilation after rest. The duration of this increase in RVD and whether or not additional benefits exist require further evaluation.

Our third important finding was that the IOP did not significantly change during the exercise and after the rest period, which is contrary to results presented for adults in previous studies showing a reduction in IOP after physical exercise ${ }^{26,27}$. However, the extent of IOP reduction has been found to vary among different types of exercises. The IOP-lowering effect of exercise seems to dissipate quickly, and this effect was very modest in most previous studies ${ }^{28}$. In our study, the children used a low-impact physical activity, cycling, for the exercise test, which did not induce a detectable reduction in IOP. We measured IOP after SS-OCT and SS-OCTA were performed, and the small delay may have weakened the exercise-induced IOP-lowering effect. Further, most children were nervous during the IOP measurement, and the anxiety may have elevated the IOP. In addition, the IOP could have been elevated if children held their breath during the cycling exercise ${ }^{29}$. All of the above factors may affect the evaluation of exercise induced IOP-lowing effect.

Some limitations to our study exist. First, we did not find an exercise protocol specifically designed for children; therefore, we relied on protocols used for adults in previous studies and basic guidelines for exercise tests ${ }^{6,20,30}$. The final exercise protocol was selected based on the performance of children in a preliminary study. Because we have recorded detailed data from this exercise protocol in children, future studies can make adjustments based on our data to meet their own needs. Second, we did not monitor systemic dynamic parameters in real time during exercise, and we evaluated exercise intensity based on the HR measured immediately after exercise. This protocol could have led us to underestimate exercise intensity. However, during the entire exercise process, a specialized researcher monitored the children and guided them to ensure that they maintained a constant pedaling speed. In addition, because SS-OCTA cannot detect blood flow velocity, a more accurate assessment of the changes in fundus blood flow was not possible.

In conclusion, moderate physical exercise for 20 min by children 9-13 years old significantly decreased the $\mathrm{CT}$ for at least $30 \mathrm{~min}$ after the exercise period and increased the fundus VD after rest. In addition, there was a significant difference of exercise induced CT thinning and RVD fluctuation between myopic and emmetropic eyes. Based on these results, we conclude that there are differences between children and adults, myopic and emmetropic eyes in the effects of exercise on fundus VD and CT. Further studies should clarify the mechanisms and effects underlying these differences. 


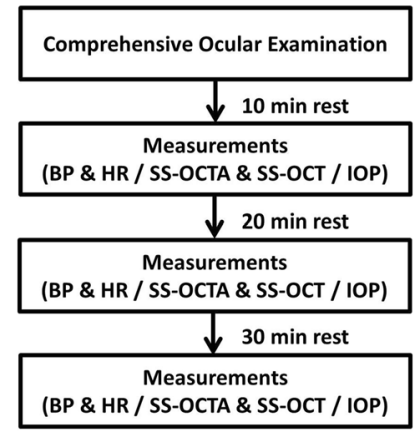

Figure 5. Flow diagram of all subjects through the study. $B P$ blood pressure; $H R$ heart rate; SS-OCTA swept source optical coherence tomography angiography; SS-OCT swept source optical coherence tomography; IOP intraocular pressure.

\begin{abstract}
Methods
Subjects and ophthalmic and systemic examinations. Forty myopic children (mean age: $11.70 \pm 1.04$ years; 23 males, 17 females) and eighteen emmetropic children (mean age: $11.06 \pm 1.35$ years; 12males, 6 females) aged 9-13 years were recruited from the Eye Hospital of Wenzhou Medical University for this study. The study was performed in accordance with the tenets of Declaration of Helsinki and was approved by the Ethics Committee of Wenzhou Medical University. Written informed consent was obtained from all the children and their parents after an explanation of the study content and methods.

Each subject underwent a comprehensive ocular examination, including slit-lamp examination, subjective refraction, AL measurement using a Lenstar LS900 (Haag-Streit AG, Koeniz, Switzerland), and intraocular pressure (IOP) measurement with a computerized tonometer (CT-1, Topcon Corporation, Tokyo, Japan). All examinations were performed in the above order. The inclusion criteria were best-corrected monocular visual acuity of at least $0.1 \log$ (minimum angle of resolution), refractive error $<+0.50 \mathrm{D}$ and $>-5.00 \mathrm{D}$, and IOP $<21 \mathrm{mmHg}$. After the ocular examination and a 10-min rest period, HR, SBP, and diastolic BP (DBP) were measured via an automated BP cuff before exercise.

The physical exercise consisted of 20 min of riding a stationary bicycle (Merach Corporation, Hangzhou, China), and was performed under the supervision of a specialized researcher. The exercise was discontinued in the event of intense discomfort. Considering that children cannot bear heavy exercise loads, the protocol was adapted from previous studies of adult cycling exercise protocols ${ }^{6}$ and American Heart Association guidelines $\mathrm{s}^{15,31}$. The exercise began with a warm-up period of 10 min during which the resistance was set at level 1 , which was equivalent to $0 \mathrm{~kg}$ of resistance. During that period, the children were encouraged to maintain a speed of $20 \mathrm{~km} / \mathrm{h}$. In the second 10-min period, the resistance level was increased to level 2 , which was equivalent to $0.5 \mathrm{~kg}$ of resistance, and the participants were encouraged to maintain a pedaling speed above $25 \mathrm{~km} / \mathrm{h}$. Handrail support was not encouraged during the cycling exercise because it may attenuate the physiological responses ${ }^{32}$. Given that heavy breathing after exercise negatively affects the quality of ocular imaging, we measured the SBP, DBP, and HR immediately within $1 \mathrm{~min}$ after the exercise was completed. We then obtained the SS-OCT and SS-OCTA images. The same measurements were repeated after a 30-min rest period (Fig. 5).
\end{abstract}

SS-OCT and SS-OCTA measurements. The subjects were imaged using a DRI-Triton SS-OCT (Topcon Corporation, Tokyo, Japan) with a central wavelength of $1,050 \mathrm{~nm}$, a scanning speed of 100,000 A-scans per second, and axial and transverse resolutions of 8 and $20 \mu \mathrm{m}$, respectively, in tissue. All SS-OCT and SS-OCTA images were acquired in follow-up mode and repeated three times by a well-trained examiner before and immediately after exercise and after the 30 -min rest period.

Macular CT was obtained with the automatic built-in software of the SS-OCT device. The SS-OCT scan was a $6 \times 6-\mathrm{mm}$ radial scan centered on the fovea, with a scan resolution of $1024 \times 12$ pixels. Thickness maps were created according to the Early Treatment Diabetic Retinopathy Study (ETDRS) grid with nine independent subfields. The 1-mm diameter central region was centered on the fovea. The parafoveal regions $(1-3 \mathrm{~mm}$ diameter, centered on the fovea) included the inner nasal, inner superior, inner temporal, and inner inferior regions. The perifoveal region (3-6 mm diameter, centered on the fovea) included the outer nasal, outer superior, outer temporal, and outer inferior regions. The SFCT was measured with a $9-\mathrm{mm}$ line scan. Because the automatic techniques to quantify CT may be influenced by variability in anatomical features, manual correction was conducted by two experienced investigators when necessary ${ }^{33,34}$.

The SS-OCTA scan area was centered on the fovea and had a $3 \times 3-\mathrm{mm}$ field of view. Only images with a signal strength index above 55 were accepted. Any image with a significant motion artifact or a double-vessel pattern was excluded. For each subject, the right eye was considered first. If the pre-exercise SS-OCTA images of the right eye were of poor quality, then the left eye was selected instead. Only three left eyes were selected in this manner.

VD was defined as the percentage area occupied by perfused blood vessels in the scanned region. RVD measurements were performed in five subfields using the two innermost circles of an ETDRS grid overlay. The reproducibility of this method was assessed in a previous study and considered acceptable ${ }^{35}$. Default automatic 


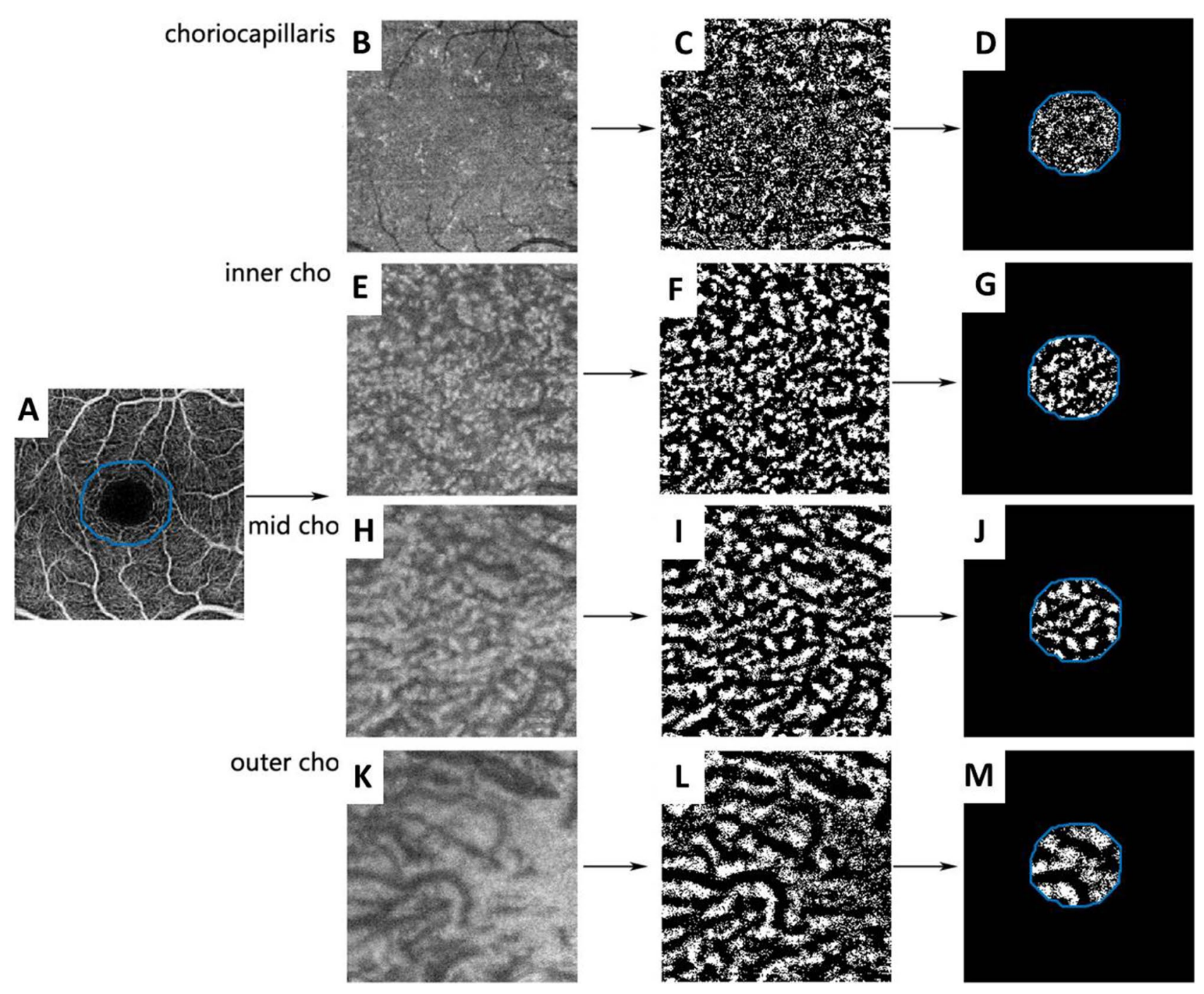

Figure 6. Representative exported and processed images. SS-OCTA and en face images were exported from the retinal superficial layer (A), choriocapillaris (B), inner choroid (inner cho, E), mid-choroid (mid cho, H), and outer choroid (outer cho, $\mathbf{K}$ ). These images were first aligned to the retinal superficial layer and then binarized in ImageJ (C, F, I, L). An area of approximately $1 / 3$ of each image, centered on the fovea, was selected to calculate choroidal vessel density (D, G, J, M). SS-OCTA swept-source optical coherence tomography.

segmentation distinguished the superficial retinal layers from $2.6 \mu \mathrm{m}$ below the ILM to $15.6 \mu \mathrm{m}$ below the IPL, while the offset for the deep retinal layers extended from 15.6 to $70.2 \mu \mathrm{m}$ below the IPL.

The CVDs of the choriocapillaris, inner choroid, mid-choroid, and outer choroid were calculated from binarized en face SS-OCTA images as described in a previous study, with some modifications ${ }^{36}$. First, we exported the retinal superficial slab and en face images of the default choriocapillaris slab $(0-10.4 \mu \mathrm{m})$. We then measured the SFCT based on each image and exported the en face images of the inner, mid-, and outer choroid at one-quarter, one-half, and three-quarters of the SFCT. Second, the three superficial retinal slabs of each subject were aligned, and an area centered on the fovea of approximately $1 / 3$ of each image was selected. Third, the choriocapillaris slab and inner, mid-, and outer choroid slabs were binarized and analyzed with MATLAB. The choroidal VD of each slab was calculated as the percentage area occupied by the choroidal vessels in the selected area (Fig. 6).

Statistical analysis. The CT, SFCT, and HR values immediately after exercise and after 30 min of rest following the exercise were normalized by subtracting the baseline (pre-exercise) values to obtain $\Delta \mathrm{CT}, \Delta \mathrm{SFCT}$, and $\Delta \mathrm{HR}$. $\Delta \mathrm{CT}$ represented the mean CT of all selected areas. Because the $\Delta \mathrm{CT}$ and $\Delta$ SFCT may be influenced by the baseline CT, we calculated the changing ratios of these parameters and obtained $\Delta \mathrm{CT} \%$ and $\Delta \mathrm{SFCT} \%$. These two parameters were analyzed with independent t-tests. The MAP was determined by the following equation: $\mathrm{MAP}=[\mathrm{SBP}+(2 \times \mathrm{DBP})] / 3^{6}$. All parameters were measured three times, and the mean values were calculated. Paired t-tests were used to analyze differences in RVD, CVD, $\triangle \mathrm{CT}, \triangle \mathrm{SFCT}, \Delta \mathrm{SBP}, \triangle \mathrm{DBP}$, and $\Delta \mathrm{HR}$ between preexercise values and the post-exercise and post-rest values. Independent $\mathrm{t}$-tests were used to analyze differences of all the measurement parameters and Chi-square test was used to analyze the difference of sex between myopic and emmetropic groups. The degrees of correlation between pairs of variables were expressed as Pearson's cor- 
relation coefficients. We performed all statistical analyses with IBM SPSS Statistics 22 for Windows (IBM Corporation, Somers, NY, USA). Unless otherwise stated, data were presented as the mean \pm standard deviation, after graphical confirmation that they met the normality assumption. For all findings, the selected level of statistical significance was a two-tailed $p<0.05$.

Received: 4 July 2020; Accepted: 11 January 2021

Published online: 21 January 2021

\section{References}

1. Khan, N. A. \& Hillman, C. H. The relation of childhood physical activity and aerobic fitness to brain function and cognition: a review. Pediatric Exerc. Sci. 26, 138-146 (2014).

2. Hillman, C. H., Khan, N. A. \& Kao, S.-C. The relationship of health behaviors to childhood cognition and brain health. Ann. Nutr. Metab. 66, 1-4 (2015).

3. Flamm, S. D. et al. Redistribution of regional and organ blood volume and effect on cardiac function in relation to upright exercise intensity in healthy human subjects. Circulation 81, 1550-1559 (1990).

4. Febbraio, M. A. Health benefits of exercise-more than meets the eye!. Nat. Rev. Endocrinol. 13, 72-74 (2017).

5. Huber, K. K., Adams, H., Remky, A. \& Arend, K. O. Retrobulbar haemodynamics and contrast sensitivity improvements after CO2 breathing. Acta Ophthalmol. Scand. 84, 481-487 (2006).

6. Vo Kim, S. et al. Optical coherence tomography angiography quantitative assessment of exercise-induced variations in retinal vascular plexa of healthy subjects. Investig. Opthalmol. Vis. Sci. 60, 1412-1419 (2019).

7. Hayashi, N., Ikemura, T. \& Someya, N. Effects of dynamic exercise and its intensity on ocular blood flow in humans. Eur. J. Appl. Physiol. 111, 2601-2606 (2011).

8. Németh, J., Knézy, K., Tapasztó, B., Kovács, R. \& Harkányi, Z. Different autoregulation response to dynamic exercise in ophthalmic and central retinal arteries: a color Doppler study in healthy subjects. Graefe's Arch. Clin. Exp. Ophthalmol. 240, 835-840 (2002).

9. Metelitsina, T. I., Grunwald, J. E., DuPont, J. C. \& Ying, G.-S. Effect of isometric exercise on choroidal blood flow in patients with age-related macular degeneration. Br. J. Ophthalmol. 94, 1629-1631 (2010).

10. Alnawaiseh, M., Lahme, L., Treder, M., Rosentreter, A. \& Eter, N. Short-term effects of exercise on optic nerve and macular perfusion measured by optical coherence tomography angiography. Retina 37, 1642-1646 (2017).

11. Alwassia, A. A. et al. Exercise-induced acute changes in systolic blood pressure do not alter choroidal thickness as measured by a portable spectral-domain optical coherence tomography device. Retina 33, 160-165 (2013).

12. Kinoshita, T. et al. Effects of exercise on the structure and circulation of choroid in normal eyes. PLoS ONE 11, e0168336 (2016).

13. Sayin, N., Kara, N., Pekel, G. \& Altinkaynak, H. Choroidal thickness changes after dynamic exercise as measured by spectral-domain optical coherence tomography. Indian J. Ophthalmol. 63, 445-450 (2015).

14. Rose, K. A. et al. Outdoor activity reduces the prevalence of myopia in children. Ophthalmology 115, 1279-1285 (2008).

15. Fletcher, G. F., Froelicher, V. F., Hartley, L. H., Haskell, W. L. \& Pollock, M. L. Exercise standards. a statement for health professionals from the American Heart Association. Circulation 82, 2286-2322 (1990).

16. Strath, S. J. et al. Guide to the assessment of physical activity: clinical and research applications. Circulation 128, 2259-2279 (2013).

17. Lacolley, P., Regnault, V., Segers, P. \& Laurent, S. Vascular smooth muscle cells and arterial stiffening: relevance in development, aging, and disease. Physiol. Rev. 97, 1555-1617 (2017).

18. Nickla, D. L. \& Wallman, J. The multifunctional choroid. Prog. Retinal Eye Res. 29, 144-168 (2010).

19. Poukens, V., Glasgow, B. J. \& Deiner, J. L. Nonvascular contractile cells in sciera and choroid of humans and monkeys. Investig. Ophthalmol. Vis. Sci. 39, 1765-1774 (1998).

20. Ikemura, T., Suzuki, K., Nakamura, N., Yada, K. \& Hayashi, N. Fluid intake restores retinal blood flow early after exhaustive exercise in healthy subjects. Eur. J. Appl. Physiol. 118, 1053-1061 (2018).

21. Zhang, S. et al. Changes in choroidal thickness and choroidal blood perfusion in guinea pig myopia. Invesigt. Ophthalmol. Vis. Sci. 60, 3074-3083 (2019).

22. Wu, H. et al. Scleral hypoxia is a target for myopia control. Proc. Natl. Acad. Sci. 115, E7091-E7100 (2018).

23. Grunwald, J. E., Riva, C. E., Stone, R. A., Keates, E. U. \& Petrig, B. L. Retinal autoregulation in open-angle glaucoma. Ophthalmol$\operatorname{ogy} 91,1690-1694(1984)$.

24. Grunwald, J. E., Riva, C. E. \& Kozart, D. M. Retinal circulation during a spontaneous rise of intraocular pressure. Br. J. Ophthalmol. 72, 754-758 (1988).

25. Kur, J., Newman, E. A. \& Chan-Ling, T. Cellular and physiological mechanisms underlying blood flow regulation in the retina and choroid in health and disease. Prog. Retinal Eye Res. 31, 377-406 (2012).

26. Najmanova, E., Pluhacek, F. \& Botek, M. Intraocular pressure response to moderate exercise during 30-min recovery. Optom. Vis. Sci. 93, 281-285 (2016).

27. Read, S. A. \& Collins, M. J. The short-term influence of exercise on axial length and intraocular pressure. Eye 25, 767-774 (2011).

28. Zhu, M. M. et al. Physical exercise and glaucoma: a review on the roles of physical exercise on intraocular pressure control, ocular blood flow regulation, neuroprotection and glaucoma-related mental health. Acta Ophthalmol. 96, e676-e691 (2018).

29. Vera, J. et al. Influence of the breathing pattern during resistance training on intraocular pressure. Eur. J. Sport Sci. 20, 157-165 (2020).

30. Lear, S. A., Brozic, A., Myers, J. N. \& Ignaszewski, A. Exercise stress testing. An overview of current guidelines. Sports Med. 27, 285-312 (1999).

31. Balady, G. J. et al. Clinician's guide to cardiopulmonary exercise testing in adults. Circulation 122, 191-225 (2010).

32. Klopfenstein Christman, S. et al. Continuous handrail support, oxygen uptake, and heart rate in women during submaximal step treadmill exercise. Res. Nurs. Health 23, 35-42 (2000).

33. Zhang, L. et al. Automated segmentation of the choroid from clinical SD-OCT. Investig. Opthalmol. Vis. Sci. 53, 7510-7519 (2012).

34. Devarajan, K. et al. Optical coherence tomography angiography for the assessment of choroidal vasculature in high myopia. $B r . J$. Ophthalmol. 2019, 314769 (2019).

35. Fernández-Vigo, J. I. et al. Reproducibility of macular and optic nerve head vessel density measurements by swept-source optical coherence tomography angiography. Eur. J. Ophthalmol. 112067211983447 (2019).

36. Wang, J. C. et al. Visualization of choriocapillaris and choroidal vasculature in healthy eyes with en face swept-source optical coherence tomography versus angiography. Transl. Vis. Sci. Technol. 7, 25 (2018).

\section{Acknowledgements}

The authors thank Dr. Britt Bromberg, Xenofile Editing (https://xenofileediting.com) for providing biomedical editing services during the final preparations of this manuscript. 


\section{Author contributions}

S.L. analysed the data and drafted this manuscript. J.B., J.X., D.P.S. and H.C. revised the manuscript detailly. S.L., Y.P., and X.L. assisted with the acquisition of the data. S.L., J.B., and H.C. designed the study. S.L. and D.P.S. made the final statistics. All authors read and approved the final manuscript.

\section{Funding}

This work was supported by the National Health Commission (wkj-zj-1707) and the National Key Research and Development Program of China (Grant No. 2016YFC0100200, 2016YFC0100201).

\section{Competing interests}

The authors declare no competing interests.

\section{Additional information}

Correspondence and requests for materials should be addressed to J.B. or H.C.

Reprints and permissions information is available at www.nature.com/reprints.

Publisher's note Springer Nature remains neutral with regard to jurisdictional claims in published maps and institutional affiliations.

(c) (i) Open Access This article is licensed under a Creative Commons Attribution 4.0 International License, which permits use, sharing, adaptation, distribution and reproduction in any medium or format, as long as you give appropriate credit to the original author(s) and the source, provide a link to the Creative Commons licence, and indicate if changes were made. The images or other third party material in this article are included in the article's Creative Commons licence, unless indicated otherwise in a credit line to the material. If material is not included in the article's Creative Commons licence and your intended use is not permitted by statutory regulation or exceeds the permitted use, you will need to obtain permission directly from the copyright holder. To view a copy of this licence, visit http://creativecommons.org/licenses/by/4.0/.

(C) The Author(s) 2021 